\title{
The Ethics of Suicide Research Online: A Consensual Protocol for Crowdsourcing- based Studies on Suicide
}

Yaakov Ophir ${ }^{1}$, Yair Amichai Hamburger², Anat Brunstein Klomek ${ }^{3}$, Yossi Levi-Belz ${ }^{4}$, Gergö Hadlaczky ${ }^{5}$, Elad Yom-Tov ${ }^{6}$, Gil Zalsman ${ }^{7}$

${ }^{1}$ Dr. Yaakov Ophir - The Faculty of Industrial Engineering and Management, Technion Israel Institute of Technology; ${ }^{2}$ Prof. Yair Amichai Hamburger - Head of The Center of Internet Psychology (CIP), the Interdisciplinary Center Herzliya; ${ }^{3}$ Prof. Anat Brunstein Klomek - Head of the Program in Clinical Psychology, Baruch Ivcher School of Psychology, The Interdisciplinary Center Herzliya; ${ }^{4}$ Prof. Yossi Levi-Belz, Head of the Lior Tsfaty Center for Suicide and Mental Pain Studies, Ruppin Academic Center, ${ }^{5}$ Prof. Gergö Hadlaczky National Prevention of Suicide and Mental Ill-Health (NASP), Karolinska Institutet, ${ }^{6}$ Dr. Elad Yom-Tov - Principal Researcher at Microsoft Research and a visiting scientist at the Technion, Israel; ${ }^{7}$ Prof. Gil Zalsman - Head of the National Council for Suicide Prevetion, Israel, Geha Mental Health Center and the Sackler school of Medicine, Tel Aviv University

Author Note: Correspondence should be addressed to Yaakov Ophir, Technion - Israel Institute of Technology. E-mail: yaakov.ophir@mail.huji.ac.il 


\begin{abstract}
Suicide research involves a difficult ethical challenge: how to keep participants' safe without compromising their privacy. This challenge becomes even more difficult in contemporary research that relies on data from anonymous users of web-based crowdsourcing platforms (e.g., Amazon's Mechanical Turk). To address this ethical challenge, a consortium of experts from multiple research institutions was assembled. The consortium discussed the advantages and disadvantages for participants involved in crowdsourcing-based studies that address suicide risk. This discussion resulted in a consensual step-by-step protocol for researchers who wish to conduct suicide research online, using the crowdsourcing platforms. This article provides a detailed description of the protocol and outlines key ethical arguments that led to its formulation. Unresolved issues are discussed as well and other researchers are encouraged to implement the proposed protocol and suggest further improvements. It is our hope that the current protocol will facilitate the research on large and diverse populations online and thus contribute to the global efforts to reduce suicide rates around the world.
\end{abstract}

Keywords: suicide research online; ethical research protocol; crowdsourcing-based research; Mechanical turk; suicide prevention 
Suicide, one of the top causes of life lost in developed countries (Abubakar, Tillmann, \& Banerjee, 2015) is a major health problem around the world (World Health Organization, 2018). As scientists and practitioners, we have a moral duty to investigate the multilayered aspects of suicide behaviors in order to improve the global efforts invested in suicide prevention (Levi-Belz, Gvion, \& Apter, 2019). Yet, the scientific research that addresses suicidal ideation and behaviors comprises a difficult ethical challenge. Assuming that researchers manage to collect clinically valid signals of suicide intentions, they face a complicated dilemma: How to keep the participants' safe without compromising their privacy. This dilemma becomes even more challenging in contemporary suicide research that is conducted online, using crowdsourcing platforms (e.g., Rogers \& Joiner, 2018; Sokol \& Eisenheim, 2016).

On the one hand the emergence of web-based crowdsourcing platforms, such as the popular forum of Amazon's Mechanical Turk (MTurk), has brought unprecedented recruitment opportunities, which may accelerate the research on suicide risk (and therefore also prevention efforts). Large, demographically diverse samples have suddenly become available for human behavior researchers (Buhrmester, Kwang, \& Gosling, 2011), including uncommon and less accessible clinical populations. Recent investigations of MTurk populations have actually showed that this platform comprises considerably high rates of clinical depression (Arditte, Çek, Shaw, \& Timpano, 2016; Walters, Christakis, \& Wright, 2018; Ophir, Sisso, Asterhan, Tikochinski \& Reichart, 2019), a substantial risk factor of suicide behaviors (Hawton \& van Heeringen, 2009). These characteristics make MTurk similar crowdsourcing platforms particularly attractive for researchers who struggle to recruit participants with suicide ideation.

On the other hand, the ethical responsibility for the safety of anonymous participants online participants who are not confined to the physical location of the researcher - seems 
extremely difficult. In comparison with offline traditional research, online researchers do not have the privilege to conduct face-to-face suicide risk assessments, and they cannot escort participants to mental health services when necessary. These challenges might discourage researchers from using crowdsourcing platforms in suicide research. Although key principles for suicide screening in mental health/educational institutions are available by the American Foundation for Suicide Prevention (AFSP, 2019), to our knowledge, there is currently a shortage of ethical and safety recommendations in a consensual protocol for researchers who wish to conduct ethical research online using crowdsourcing platforms.

To address this ethical challenge, we assembled a consortium of experts in suicide and Internet-based research from a variety of research institutions around the world. In the past year, we conducted thorough discussions (both in writing and in conference calls) on the advantages and risks of crowdsourcing-based suicide research. This process yielded a stepby-step protocol for ethical research online. In this document, we present the proposed protocol in a detailed and concrete manner. We then outline five unresolved ethical issues for future scientific and clinical discussions. Finally, we discuss key ethical arguments that led to the formulation of the current version of the protocol.

\section{A step-by-step protocol for online crowdsourcing-based suicide research}

1. As in any other non-suicide related research, participants in crowdsourcing-based suicide research will be asked to sign an informed consent form, which includes a clear and detailed description of the study's goals and procedure. However, unlike less sensitive research, suicide researchers should make additional effort that participants will read the full consent form. It is therefore advised to implement attention checks by the end of the consent form. Attention checks for example can include a verbal question with only one correct answer regarding the content of the consent form (for a comprehensive list of attention checks, see in Ophir et al., 2019). Participants who fail either one of these 
attention checks should receive a note that they are kindly requested to dedicate one more minute to read the full form.

2. Participants will be assured that their answers will remain confidential and that their data will be used for research and for their safety only. Through the consent form, participants will also be informed that their answers will be monitored and that they might be approached by the research team through the crowdsourcing system if their answers would imply that they are at risk. A template for this statement can be: "Dear participant, please note that the answers to this survey are monitored by the principle investigators. This is because your answers may contain references to extremely distressing feelings or to thoughts about suicide. You may receive a message from us in which we will encourage you to seek professional help and refer you to a list of services and hotlines that are helpful for coping with distressing feelings. Please be sure that this procedure does not expose your name or contact details."

3. Crowdsourcing platforms allow researchers to set the specific locations from which participants would be recruited. Local contact information of mental health and suicide prevention hotlines should be presented throughout the data collection procedure, according to the chosen locations. It is the principle researchers' responsibility to collect contact details of mental health services that are in the same geographical location of the participants.

4. It is recommended that suicide ideation and behaviors will be measured using wellestablished, validated suicide scales such as the Columbia Suicide Severity Rating Scale (Posner et al., 2011), the Suicide Attempt Self-Injury Interview (Linehan, Comtois, Brown, Heard \& Wagner, 2006), or the Suicidal Behaviors Questionnaire-Revised (Osman, Bagge, Gutierrez, Konick, Kopper, \& Barrios, 2001). It is also recommended to 
use scales with validated online versions (i.e. that were previously administered to participants online (e.g., Viguera et al., 2015).

5. It is recommended that an open-ended item be added following the closed-suicide scale to allow participants to share and elaborate their distressed feelings. The open-ended question may encourage participants to share their authentic experiences and provide them an opportunity to unload their emotional distress in free writing. In our experience, participants appreciate the opportunity to be heard, even if they remain anonymous. A template for this open-ended item may be: "Do you have any verbal comments regarding the previous questions (that addressed suicide ideation/behaviors)? You are more than welcome to share your feelings with us".

6. Upon completion of the study, participants' answers to the suicide questionnaire will be computed. This can be done using any data analysis software (e.g., Microsoft Excel, Qualtrics). Researchers should also inspect the open-ended item manually and mark participants who stated that they contemplate suicide. Participants, who are marked with potential suicide risk in either method, should receive a designated letter signed personally by the principle investigator. In light of the unique characteristics of crowdsourcing, any indication for suicide risk including "low levels" of suicidal thoughts should be addressed with this designated letter. Although many individuals with suicidal thoughts may not be at a significant risk, clinicians are recommended to evaluate these thoughts as well (Gliatto \& Rai, 1999). The letter will be sent through the crowdsourcing system without the need to ask participants for their contact details. Crowdsourcing platforms usually assign serial numbers to their users so their real names and contact information remain confidential. Unlike traditional offline studies, in crowdsourcingbased studies researchers can contact participants through the website interface without compromising the privacy of the participants, thus avoiding the usual "trade-off" between 
privacy and safety. If a crowdsourcing platform does not allow the researcher to contact participants after the study, we do not recommend using it for suicide research.

7. The letter to participants at risk will include a list of mental health centers along with web-links and telephone numbers of counselling services. Mental health services that are suspected to be inadequate should be excluded from this list. The designated letter will also include an explicit encouragement for participants to seek help and contact their local mental health services. A template for the designated letter to participants at risk is presented in the Appendix.

8. Some participants might respond to this letter, either through the crowdsourcing system or through a direct communication with the principal investigators. Any reaction from participants should be personally and respectfully answered by the principle investigator or by her/his qualified representative. The respondent professional background should include relevant training and experience in mental health counseling. We encourage researchers to dedicate the time to match the response to the specific content raised by the participant. Yet, we offer here an example for a general response: "Dear participant, thank you very much for sharing this with us. The experiences you describe are indeed very challenging. We do however believe that therapy may help you feel better and much more in control. We wish that you would always have hope and we encourage you once again to seek professional help". The aim of this response (following participants' reactions to the designated letter) is not to replace professional therapy but to help participants feel heard and respected and to keep encouraging them to turn to local mental health services.

\section{Unresolved ethical issues for further discussion}

A. The current protocol addresses the ethical aspects of suicide research. It does not discuss legal liabilities. Needless to say that research is subjected to the law in each state. We recommend that researchers consult with their local Institutional Review Board (IRB) 
regarding the specific laws in their country. To our knowledge, the legal condition of this emerging field is still unclear. A similar topic is known in the medical literature as "unsolicited diagnosis" (Mitchell, 2011). How for example should dermatologists react when they randomly notice a suspicious mole on the person standing in front of them in a crowded elevator (Preller \& Salloch, 2018)? The ethical stance that is manifested in the current protocol is that researchers should not offer participants unsolicited formal DSMbased diagnoses (e.g., Major Depressive Disorder) but that they are required to contact participants in life threating situations and encourage them to seek help.

B. The proposed consent form includes a "trigger" warning that the upcoming research involves personal questions that address sensitive topics, such as depression and suicide ideation. We acknowledge that this warning might cause a bias in the recruited sample. This warning might alienate potential participants from participating in the research despite the promise for anonymity, especially if they do not feel comfortable sharing sensitive information about themselves.

C. The current protocol includes a specific recommendation to include an open-ended research item in which participants could share their emotions in an unrestricted or expansive manner. As mentioned above, the rationale behind this recommendation is twofold: to encourage participants to share their authentic experiences and to provide them the opportunity to unload their emotional distress. However, this item requires a manual inspection, which might burden researchers, especially those investigating large samples. Moreover, participants might leverage this open-ended item to manifest a concrete and immediate will to die by suicide. It is arguable that an explicit suicide manifestation requires the researcher to communicate the threat to local law enforcements. Yet, since the contact information of the participants is concealed, this act could be impossible. 
D. Another recommendation of the current guidelines is to provide participants with local contact information of mental health and suicide prevention hotlines. However, despite the fact that crowdsourcing platforms allow researchers to set their location priorities, participants can be physically in a different location. De facto, they can complete the research questionnaires, while they are traveling abroad. Researchers may consider more rigid inclusion criteria in which crowdsourcing users could only participate in research if they declare that they are completing the questionnaire from the requested location.

Alternatively, researchers can limit the participation to Internet Protocol (IP) addresses that are associated with the requested location.

E. The current version of the protocol relies on external resources, such as the 24/7 National Suicide Prevention Lifeline (see Appendix), which create an unbalanced burden on these governmental/non-profit organizations. In order to share this burden, suicide researches may consider allocating economical or human resources to compensate these organizations. Future discussions can determine the nature of this relationship between researchers and prevention organization and whether it should be based on more formal contracts.

\section{Summary and key arguments}

This document presented a consensual step-by-step protocol for the safe implementation of crowdsourcing-based research on suicide. The protocol resulted from a cooperative work of suicide and Internet researchers from multiple countries. We acknowledge that the final protocol may be somewhat limited and we hope that other suicide researchers who would implement the current protocol would provide more suggestions for improvements, based on their accumulated experience in crowdsourcing-based research. Following are some of the key arguments that lay the ethical foundation for the development the current protocol. 
Survey-based suicide research (without a specific research manipulation) does not put participants at risk. Although suicide questionnaires may raise a certain degree of discomfort, they do not increase actual suicide behaviors (Dazzi, Gribble, Wessely, \& Fear, 2014). This means that a main risk for participants in suicide research is the violation of their privacy. Yet, specifically in crowdsourcing-based research, the privacy of the participants is not compromised. Indeed, some participants with suicide ideation might be left without proper treatment in online crowdsourcing-based research. Yet, this unfortunate situation is not created by the research setting. The only real negative outcome for the participant is the fact that the researcher knows that someone (i.e., the participant who is shown only by his/her serial number) suffers from suicide ideations.

Not only does crowdsourcing-based research online not violate participants' privacy, participants may actually benefit from such research (Dazzi et al., 2014). Participants can use the survey, and especially the open-ended item, as an outlet for their emotional pain and they can be motivated to turn to therapy, following the letter they received from the researches. Through the online research, individuals with suicide ideation receive an opportunity to voice their emotional difficulties and accept a sensitive letter that acknowledges their pain and perhaps even helps them overcome barriers in treatment seeking. Thus, it is possible that crowdsourcing users' situations may improve compared with individuals who do not participate in the research.

Considering the minimal risk and the potential benefit for online participants, we encourage researchers to leverage the accessibility to large and diverse samples in crowdsourcing platforms for suicide research. Suicide, as mentioned previously, is a major health problem and together with depression it constitutes a great psychiatric and economic burden in developed countries (Abubakar et al., 2015; World Health Organization, 2018; Greenberg, Fournier, Sisitsky, Pike, \& Kessler, 2015). Speaking in the name of all the 
consortium members, we hope that the current protocol will remove barriers facing researchers who wish to conduct sensitive surveys online and that it will ultimately accelerate studies on suicide risks and contribute to the global efforts to reduce suicide around the world. 


\section{Appendix - A template for a letter to participants}

Dear participant,

Thank you for completing the survey on [Name of online crowdsourcing platform] as part of the research [Name of research]. We appreciate your openness and take your responses very seriously.

Our automatic system has indicated that you might be feeling very upset and overwhelmed by what is happening in your life. We understand that feeling suicidal may be a part of feeling overwhelmed.

You are not alone. We care about you and we believe that there are other better solutions, treatment that can relieve your pain and help you to not harm yourself. We encourage you to seek help through your local mental health services.

[RESEARCHER TO INSERT LOCAL MENTAL HEALTH SERVICES]

$\underline{\text { A List for example of 24/7 first aid mental health services }}$

- The National Suicide Prevention Lifeline: 1-800-273-TALK (8255)

- An online emotional support chat, which is available 24/7 across the U.S: http://www.suicidepreventionlifeline.org/gethelp/lifelinechat.aspx

- Crisis Text Line: For a free, 24/7 support from a trained crisis counselor, please text 741741 from anywhere in the US.

- For any emergency you can also call 911 in the US or 112 in the EU.

- Last, please see this list of international emergency phone numbers: https://en.wikipedia.org/wiki/List_of_emergency_telephone_numbers

Best wishes,

[Name of principle researcher, including practitioner title], on behalf of the [Name of research'] research team

[Email address of the PI] 


\section{Bibliography}

Abubakar, II, Tillmann, T., \& Banerjee, A. (2015). Global, regional, and national age-sex specific all-cause and cause-specific mortality for 240 causes of death, 1990-2013: a systematic analysis for the Global Burden of Disease Study 2013. Lancet, 385(9963), 117-171. doi: 10.1016/S0140-6736(14)61682-2.

AFSP (2019). Interactive Screening Program. American Foundation for Suicide Prevention. Last retrieved on July 14, 2019, from https://afsp.org/our-work/interactive-screeningprogram/

Arditte, K. A., Çek, D., Shaw, A. M., \& Timpano, K. R. (2016). The importance of assessing clinical phenomena in Mechanical Turk research. Psychological Assessment, 28(6), 684. doi: 10.1037/pas0000217.

Dazzi, T., Gribble, R., Wessely, S., \& Fear, N. T. (2014). Does asking about suicide and related behaviours induce suicidal ideation? What is the evidence? Psychological Medicine, 44(16), 3361-3363. doi: 10.1017/S0033291714001299

Gliatto, M. F., \& Rai, A. K. (1999). Evaluation and treatment of patients with suicidal ideation. American family physician, 59(6), 1500-1506. doi: unavailable

Greenberg, P. E., Fournier, A.-A., Sisitsky, T., Pike, C. T., \& Kessler, R. C. (2015). The economic burden of adults with major depressive disorder in the United States (2005 and 2010). J Clin Psychiatry, 76(2), 155-162. doi: 10.4088/JCP.14m09298.

Hawton, K., \& van Heeringen, K. (2009). Suicide. The Lancet, 373(9672), 1372-1381. doi:10.1016/S0140-6736(09)60372-X

Mitchell, E. W. (2011). The ethics of unsolicited diagnosis of mental disorder in acquaintances: benefits and dangers. The Psychiatrist, 35(8), 297-301. doi:10.1192/pb.bp.110.032953

Levi-Belz Y, Gvion Y and Apter A (2019) Editorial: The Psychology of Suicide: From 
Research Understandings to Intervention and Treatment. Front. Psychiatry 10:214. doi: 10.3389/fpsyt.2019.00214

Linehan, M. M., Comtois, K. A., Brown, M. Z., Heard, H. L., \& Wagner, A. (2006). Suicide Attempt Self-Injury Interview (SASII): development, reliability, and validity of a scale to assess suicide attempts and intentional self-injury. Psychological assessment, 18(3), 303. doi: 10.1037/1040-3590.18.3.303.

Ophir, Y., Sisso, I., Asterhan, C. S. C., Tikochinski, R., \& Reichart, R. (2019). The Turker Blues: Hidden Factors Behind Increased Depression Rates Among Amazon's Mechanical Turkers. Clinical Psychological Science, 2167702619865973. doi:10.1177/2167702619865973

Osman, A., Bagge, C. L., Gutierrez, P. M., Konick, L. C., Kopper, B. A., \& Barrios, F. X. (2001). The Suicidal Behaviors Questionnaire-Revised (SBQ-R): validation with clinical and nonclinical samples. Assessment, 8(4), 443-454. doi: 10.1177\%2F107319110100800409.

Posner, K., Brown, G. K., Stanley, B., Brent, D. A., Yershova, K. V., Oquendo, M. A., . . . Shen, S. (2011). The Columbia-Suicide Severity Rating Scale: initial validity and internal consistency findings from three multisite studies with adolescents and adults. American Journal of Psychiatry, 168(12), 1266-1277. doi:

10.1176/appi.ajp.2011.10111704.

Preller, G., \& Salloch, S. (2018). Melanoma in the shopping mall: A utilitarian argument for offering unsolicited medical opinions in informal settings. Bioethics, 32(3), 193-198. doi: 10.1111/bioe.12426.

Rogers, M. L., \& Joiner, T. E. (2018). Suicide-Specific Rumination Relates to lifetime suicide attempts above and beyond a variety of other suicide risk factors. Journal of Psychiatric Research, 98, 78-86. doi: 10.1016/j.jpsychires.2017.12.017 
Sokol, Y., \& Eisenheim, E. (2016). The Relationship Between Continuous Identity Disturbances, Negative Mood, and Suicidal Ideation. The primary care companion for CNS disorders, 18(1), 10.4088/PCC.4015m01824. doi:10.4088/PCC.15m01824

Viguera, A. C., Milano, N., Laurel, R., Thompson, N. R., Griffith, S. D., Baldessarini, R. J., \& Katzan, I. L. (2015). Comparison of electronic screening for suicidal risk with the Patient Health Questionnaire Item 9 and the Columbia Suicide Severity Rating Scale in an outpatient psychiatric clinic. Psychosomatics, 56(5), 460-469. doi: 10.1016/j.psym.2015.04.005.

Walters, K., Christakis, D. A., \& Wright, D. R. (2018). Are Mechanical Turk worker samples representative of health status and health behaviors in the US? PloS one, 13(6), e0198835. doi: 10.1371/journal.pone.0198835.

World Health Organization (2018). Suicide. Last retrieved on October 9, 2019, from https://www.who.int/news-room/fact-sheets/detail/suicide 